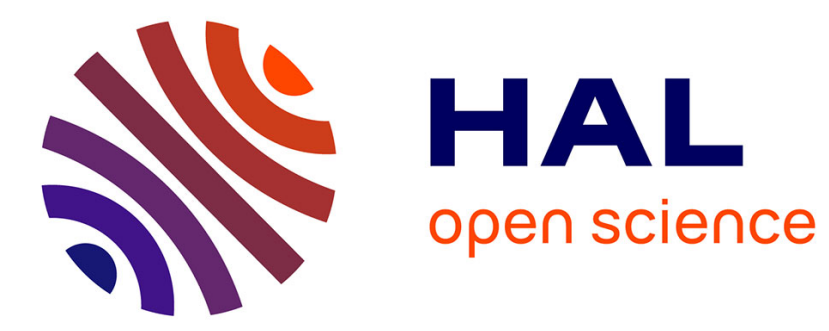

\title{
Existence and computation of Berge equilibrium and of two refinements
}

Pierre Courtois, Rabia Nessah, Tarik Tazdaït

\section{To cite this version:}

Pierre Courtois, Rabia Nessah, Tarik Tazdaït. Existence and computation of Berge equilibrium and of two refinements. Journal of Mathematical Economics, 2017, 72, pp.7-15. 10.1016/j.jmateco.2017.04.004 . hal-01607922

\section{HAL Id: hal-01607922 \\ https://hal.science/hal-01607922}

Submitted on 26 May 2020

HAL is a multi-disciplinary open access archive for the deposit and dissemination of scientific research documents, whether they are published or not. The documents may come from teaching and research institutions in France or abroad, or from public or private research centers.
L'archive ouverte pluridisciplinaire HAL, est destinée au dépôt et à la diffusion de documents scientifiques de niveau recherche, publiés ou non, émanant des établissements d'enseignement et de recherche français ou étrangers, des laboratoires publics ou privés.

\section{다(1)(2)}

Distributed under a Creative Commons Attribution - ShareAlikel 4.0 International 


\title{
Existence and Computation of Berge Equilibrium and of two refinements
}

\author{
Pierre Courtois* $^{*} \quad$ Rabia Nessah ${ }^{\dagger} \quad$ Tarik Tazdaït
}

April, 2017

\begin{abstract}
This paper presents existence conditions as well as computation methods for Berge equilibrium and two refinements: Berge-Vaisman equilibrium and Berge-Nash equilibrium. Each equilibrium concept is interpreted and illustrated on the basis of relevant examples and general existence conditions satisfying weak continuity and quasi-concavity conditions are provided.

Keywords: Berge equilibrium, individual rationality, diagonal transfer quasi-concavity, diagonal transfer continuity.
\end{abstract}

\section{Introduction}

Berge equilibrium (BE) (Berge 1957, Zhukovskii and Chikrii 1994) and its refinements (Vaisman 1994, 1995, Abalo and Kostreva 1996, 2004) have attracted increasing attention recently in the game theoretic literature for its theoretical contributions to the modeling of social norms and prosocial behaviors. Colman et al. (2011) define and interpret BE as a mutual support equilibrium. In playing $\mathrm{BE}$, agents support each other by mutually selecting actions that maximize the welfare of others. The assumption is that they adopt such behavioral norms because of the reciprocal dimension implied. Courtois et al. (2015) develop a situational theory in which players follow either a Berge or Nash behavior rule. The hypothesis is that agents adopt the behavior rule that is the most beneficial to them. This means that in social situations such as trust games or social dilemmas, players would mutually support each others, while in competitive situations such as zero sum games, they would simply maximize their welfare independently from the welfare of others.

A key criticism of this theory is that $\mathrm{BE}$ is not immune to unilateral deviation. It is not either necessarily compatible with individual rationality. Two refinements of the concept address partly these issues. The Berge-Vaisman equilibrium (BVE) (Vaisman 1994, 1995), restricts the set of

\footnotetext{
*LAMETA, INRA, Supagro, CNRS, Montpellier Univ., 34000 Montpellier, France. contact: pierre.courtois@ @inra.fr

${ }^{\dagger}$ IESEG School of Management, LEM, CNRS, Lille, France. contact: r.nessah@ ieseg.fr

${ }^{\ddagger}$ CIRED, CNRS, EHESS, Ecole des Ponts, Nogent sur Marne, France. contact: tazdait@ centre-cired.f
} 
$\mathrm{BE}$ to the subset in which the equilibrium gain of each agent is no lower than his maximin payoff. BVE precludes all BE that are not individually rational (see Crettez (2016) for sufficient conditions for BE to be BVE). Another refinement defined by Abalo and Kostreva $(1996,2004)$ goes further and restricts the set of $\mathrm{BE}$ to the subset in which Berge strategy is a best reply. Berge-Nash equilibrium (BNE) combines the properties of Berge and of Nash equilibrium (NE) and admits the advantage of characterizing the set of BE that are incentive compatible.

The current paper offers general existence conditions as well as computation methods for BE (in coalitional or individualistic form), BVE, and BNE. Up until now, existence results have only been proposed for BE in coalitional form (CBE) and for BNE for n-player games in which (1) the strategy spaces are nonempty, convex, and compact, and (2) players have continuous and quasiconcave payoff functions (Nessah et al. 2007, Larbani and Nessah 2008). Existence results for BVE have also been proposed, but these results are limited to the case of differential games with quadratic payoffs (Vaisman 1994, 1995, Zhukovskii and Chikrii 1994). Nessah and Larbani (2014) gave a general existence result that weakens the concavity and continuity conditions for BE in individualistic form (also called Berge-Zhukovskii equilibrium (BZE)) in 2-player games. Generalizing these results, this paper investigates new existence conditions for CBE, BNE, and BVE in n-player games that satisfy weak continuity and quasi-concavity conditions. The proposed existence conditions for the three equilibrium concepts are theoretically related and based on the notions of diagonal transfer quasi-concavity and diagonal transfer continuity (Baye et al. 1993). The two notions are weak concepts of quasi-concavity and continuity which adopt a basic idea of transferring a set of strategy profile(s) to another set of strategy profile(s). The key contribution of the paper is therefore to provide simple existence and computation methods conditions that allow for BVE, $\mathrm{BNE}, \mathrm{BZE}$ and $\mathrm{CBE}$ to be used in the largest possible class of games.

The paper is organized as follows. In Section 2, we define CBE, BZE, BVE and BNE and interpret these equilibrium concepts on the basis of several examples. In Section 3, we provide the sufficient conditions for their existence, and we derive operational procedures for their computation Section 4 concludes and makes suggestions for further research concerning this topic.

\section{Definitions and Interpretations}

Consider the game

$$
G=\left(X_{i}, u_{i}\right)_{i \in I}
$$

where $I=\{1,2, \ldots, n\}$ is the set of players, $X=\prod_{i \in I} X_{i}$ is the set of strategy profiles in the game, $X_{i}$ is the set of strategies of player $i, X_{i} \subset E_{i}, E_{i}$ is a vector space, and $u_{i}: X \longrightarrow \mathbb{R}$ is the bounded payoff function of player $i$.

Let $\Im$ denote the set of all coalitions (i.e., nonempty subsets of $I$ ). For each coalition $C \in \Im$, 
we have a complementary coalition set denoted by $-C$. If $C$ is reduced to a singleton $\{i\}$, the set $-C$ is denoted by $-i$. We also denote $X_{C}=\prod_{i \in C} X_{i}$ as the set of strategies of the players in coalition $C$. Let $R=\left\{R_{i}\right\}_{i \in M}$ be a partition of the set of players $I$ where $M=\{1, \ldots, s\}$ is an index set. Any strategy profile $x=\left(x_{1}, \ldots, x_{n}\right) \in X$ can be written $x=\left(x_{R_{1}}, x_{R_{2}}, . ., x_{R_{s}}\right)$.

We start with a definition of CBE as introduced in Berge (1957, p. 88-89).

Definition 2.1 (Berge (1957)) Consider the game (2.1) and let $R=\left\{R_{i}\right\}_{i \in M} \subset \Im$ be a partition of $I$ and $S=\left\{S_{i}\right\}_{i \in M}$ be a set of subsets of $I$. A feasible strategy $\bar{x} \in X$ is an equilibrium point for the set $R$ relative to the set $S$ or a coalitional Berge equilibrium (CBE) for (2.1) if

$$
u_{r_{m}}(\bar{x}) \geq u_{r_{m}}\left(\bar{x}_{-S_{m}}, x_{S_{m}}\right) \text {, for each given } m \in M, \text { any } r_{m} \in R_{m}, \text { and } x_{S_{m}} \in X_{S_{m}} .
$$

A strategy profile $\bar{x}$ is a CBE if no player in any coalition $R_{m}$ in $R$, can be better off when the players in the corresponding coalition $S_{m}$ in $S$, deviate from their BE strategy profile $\bar{x}_{S_{m}}$. This means that at CBE, the players in coalition $S_{m}$ play a strategy profile that maximizes the payoff of the players in coalition $R_{m}$, but they neglect or ignore their own payoffs (when $S_{m} \bigcap R_{m}=\emptyset$ ). At CBE the payoffs of the players in $S_{m}$ are taken care of by other players, making this rule of conduct resemble a reciprocal behavior. This equilibrium concept could be summed up by the following perspective: "I care about your welfare because you care about mine making both of us better off".

EXAMPLE 2.1 (Climate Coalitional Game) Consider an economy consisting of $n$ countries. Let $I=\{1,2, \ldots, n\}$ be the index set of countries, $R=\left\{R_{1}, \ldots, R_{s}\right\}$ be a partition of $I$ and $S=$ $\left\{S_{1}, \ldots, S_{s}\right\}$ be a coalition structure.

Let $e_{i} \geq 0$ and $q_{i}=g_{i}\left(e_{i}\right)$ denote the emission level and the output resulting from this emission level for country $i$. Let $z\left(e_{1}, \ldots, e_{n}\right)=\sum_{i \in I} e_{i}$ be the total pollution level and $v_{i}(z)$ be country's $i$ disutility resulting from this pollution. The net utility of player $i$ is then

$$
u_{i}\left(e_{1}, \ldots, e_{n}\right)=g_{i}\left(e_{i}\right)-v_{i}\left(z\left(e_{1}, \ldots, e_{n}\right)\right) .
$$

For each coalition $R_{m}, m=1, \ldots, s$, define the utility of any country in this coalition as follows:

$$
\widetilde{U}_{h}\left(e_{1}, \ldots, e_{n}\right)=\sum_{j \in R_{m}} u_{j}\left(e_{1}, \ldots, e_{n}\right), \text { for each } h \in R_{m}
$$

Then for any given $(R, S)$, we associate a game $\left(X_{i}, \widetilde{U}_{i}, R, S\right)_{i \in I}$. Consider $n=4, g_{i}\left(e_{i}\right)=$ $\alpha_{i} \sqrt{e_{i}}, v_{i}(z)=\beta_{i} z+\gamma_{i}$, for all $i=1,2,3,4$ and assume that $R_{m}=S_{m}$, for each $m=1, \ldots, s$.

If $R=\{\{1,2\},\{3,4\}\}$, the unique $\mathrm{CBE}$ is given by

$$
\bar{e}_{i}=\frac{\alpha_{i}^{2}}{4\left(\beta_{1}+\beta_{2}\right)^{2}}, i=1,2 \text { and }
$$




$$
\bar{e}_{i}=\frac{\alpha_{i}^{2}}{4\left(\beta_{3}+\beta_{4}\right)^{2}}, i=3,4
$$

If $R=\{\{1,2,3\},\{4\}\}$, the unique $\mathrm{CBE}$ is given by

$$
\bar{e}_{i}=\frac{\alpha_{i}^{2}}{4\left(\beta_{1}+\beta_{2}+\beta_{3}\right)^{2}}, i=1, \ldots, 3 \text { and } \bar{e}_{4}=\frac{\alpha_{4}^{2}}{4 \beta_{4}^{2}} .
$$

If $R=\{\{1,2,3,4\}\}$, the unique $\mathrm{CBE}$ is given by

$$
\bar{e}_{i}=\frac{\alpha_{i}^{2}}{4\left(\beta_{1}+\beta_{2}+\beta_{3}+\beta_{4}\right)^{2}}, i=1, \ldots, 4
$$

If we let $j \in S_{m}$, and since the family of coalitions $R$ is a partition of the set of players $I$, there exists some $p \in M$ such that $j \in R_{p}$. According to the definition of CBE, the players of the corresponding coalition $S_{p}$ maximize the payoff functions of the players in $R_{p}$, and since $j \in R_{p}$, the payoff of player $j$ is also maximized by the players of $S_{p}$. We deduce that at a CBE, each player maximizes the payoff of at least one of the other players and in turn, his own payoff is maximized by at least one other player. Reformulating this coalitional equilibrium concept from an individualistic perspective, we obtain what we call a BZE as defined by Zhukovskii (1985).

DEFINITION 2.2 (Zhukovskii (1985)) A strategy profile $\bar{x} \in X$ is a Berge-Zhukovskii equilibrium (BZE) of the game (2.1) if

$$
u_{i}(\bar{x}) \geq u_{i}\left(\bar{x}_{i}, y_{-i}\right) \text {, for each, given } i \in I \text { and } y_{-i} \in X_{-i} \text {. }
$$

In order to show that BZE is a special case of CBE, assume that $M=I, R_{i}=\{i\}, i \in I$, $S_{i}=-i$, and $i \in I$. This means that when playing the BZE strategy $\bar{x}_{i}$, a player $i \in I$ yields his highest possible utility when other players also play according to their BZE strategy. This also means that this same player, when following strategy $\bar{x}_{i}$, cannot obtain a maximum payoff unless the remaining players $-i$ are willingly to play the strategy $\bar{x}_{-i}$. We deduce that if all players play $\bar{x}$, then all payoffs are maximized but if at least one of the players $j$ deviates from his equilibrium strategy, then the payoff of any player $i$ in $-j$, the resulting strategy profile, is at most equal to his payoff $u_{i}(\bar{x})$ in the resulting profile.

EXAMPLE 2.2 (Costly contribution game) Consider a three players contribution game in which each player can either contribute to a collective action or retract his contribution. Set $I=3$ as the number of players and let $X_{i}=[-1,1]$ be the strategy space of player's $i$, with $i=1,2,3$. Let us first consider a symmetric payoff function such that:

$$
u_{i}(x)=-x_{i}+\sum_{j \in-i} x_{j}, i=1,2,3 .
$$


We can easily see that $\bar{x} \in X$ is a BZE if and only if $\max _{y_{-i} \in X_{-i}} u_{i}\left(\bar{x}_{i}, y_{-i}\right)=u_{i}(\bar{x})$, for any $i=$ $1,2,3$. We deduce that $\bar{x}=(1,1,1)$ is the unique pure strategy BZE of this game and $u_{i}(\bar{x})=1$, for all $i=1,2,3$. Notice that by playing BZE, each player ends up better off than by playing Nash equilibrium (NE). The unique pure strategy NE of this game is indeed $\underline{x}=(-1,-1,-1)$ and $u_{i}(\underline{x})=-1$, for all $i=1,2,3$. When all players support each other, contributions as well as players welfares are maximized.

Slightly modifying the payoff function of this game reveals that this result does not always hold. Suppose now that players have a payoff function such that:

$$
u_{i}(x)=-i x_{i}+\sum_{j \in-i} x_{j}, i=1,2,3
$$

Again, $\bar{x}=(1,1,1)$ is the unique pure strategy BZE of this game and $\underline{x}=(-1,-1,-1)$ is the unique pure strategy NE. Associated payoffs are now $u_{i}(\bar{x})=2-i$ and $u_{i}(\underline{x})=i-2$, for all $i=1,2,3$. Social welfare remains the same playing BZE or NE, but the distribution of welfare changes, causing some players to end up worse off when players play BZE. Notice also that in this case the BZE outcome is not individually rational, as some players obtain less than their security level defined by $\alpha_{i}=\max _{x_{i} \in X_{i}} \min _{y_{-i} \in X_{-i}} u_{i}\left(x_{i}, y_{-i}\right), i=1,2,3$. We have indeed $\alpha_{i}=i-2, i=1,2,3$ and $\alpha_{3}=1>-1=u_{3}(\bar{x})$.

If the contribution cost is greater, every player may end up worse off playing BZE than NE eventually decreasing social welfare. This is the case, for example, when the payoff function is such that:

$$
u_{i}(x)=-3 x_{i}+\sum_{j \in-i} x_{j}, i=1,2,3
$$

In this case, players receive $u_{i}(\bar{x})=-1$ and $u_{i}(\underline{x})=1$, for all $i=1,2,3$ respectively. Again, the unique BZE of the game is not individually rational.

In order for $\mathrm{BE}$ in a coalitional or individualistic form to be compatible with welfare maximisation requirements, and in order to preclude mutual support when BE is not individually rational, a first refinement adds an individual rationality constraint to the concept. A BE is individually rational if players receive a payoff that is at least equal to their security or maximin level (Zhukovskii and Chikrii (1994)). ${ }^{1}$ If we return to the contribution game depicted above, this is not the case in ( 2.4) and in (2.5), that is, in situations where players are either better off by playing selfishly or where some players end up worse off by playing BE. Adding individual rationality requirements allows us to discard equilibrium points where for at least one player, resulting payoff is dominated by the maximin payoff.

\footnotetext{
${ }^{1}$ In the following we define Berge-Vaisman equilibrium in an individualistic form as in Zhukovskii and Chikrii (1994). A related definition can easily be derived for BVE in coalitional form.
} 
Definition 2.3 (Zhukovskii and Chikrii (1994)) A strategy profile $\bar{x} \in X$ is a Berge-Vaisman equilibrium (BVE) of the game (2.1) if

1) $\forall i \in I, \forall y_{-i} \in X_{-i}, u_{i}(\bar{x}) \geq u_{i}\left(\bar{x}_{i}, y_{-i}\right)$,

2) $\forall i \in I, \forall y_{-i} \in X_{-i}, u_{i}(\bar{x}) \geq \alpha_{i}=\sup _{x_{i} \in X_{i} y_{-i} \in X_{-i}} u_{i}\left(x_{i}, y_{-i}\right)$.

The first condition means that BVE is a BZE (see Definition 2.2). The second condition of Definition 2.3 means that the strategy profile $\bar{x}$ is individually rational meaning that for each player $i \in I, \mathrm{BVE} \bar{x}$ yields a payoff that is greater than or equal to his security level, denoted $\alpha_{i}$.

Considering well-known games, notice that when the unique BZE in a normal form prisoner's dilemma and chicken game is individually rational, BVE and BZE coincide. The same result is obtained in the trust game defined by Kreps (1990, p.100-101) and the stag hunt game introduced by Rousseau (1964 [1755], p.167). Bertrand oligopoly, n-players public good games and n-player electoral competition games admit also a BVE. ${ }^{2}$ Conversely, in two player zero sum games, if a BZE exists, this is not a BVE, meaning that it is not individually rational to be mutually supportive. Conjecturing that situational decision-making is first driven by individual rationality, this would mean that in situations where no BVE exists, mutual support is discarded in favor of NE. Conversely, if a BVE exists players will play it.

Although less compelling from the perspective of building a situational theory of games, a further step in refining the BE concept is to add strategic rationality. As for BE in coalitional or in individualistic form, BVE is not immune from unilateral or collective deviations. In order to have a BE such that no player can do better by unilaterally changing his or her strategy, this equilibrium must also be a NE. A second refinement initially defined by Abalo and Kostreva $(1996,2004)$ merges the properties of Berge and Nash equilibrium.

DeFinition 2.4 (Abalo and Kostreva (2004)) A BE which is also a NE is called Berge-Nash equilibrium. (BNE)

This equilibrium concept is based on the conjunction of BE, BVE, and NE. It can be considered in a coalitional or in an individualistic form. BNE is a refinement of BE, and this is also a refinement of NE. Typically, such equilibrium exists in situations where interests are well aligned and where maximizing selfishly individual utility or being mutually supportive both lead to similar outcomes.

EXAMPLE 2.3 (non-costly contribution game) Let us once more consider a contribution game, but let us assume that contribution is now non-costly. As before, set $I=3$ as the number of

\footnotetext{
${ }^{2}$ Examples are available upon request.
} 
players, and let $X_{i}=[-1,1]$ be the strategy space of player $i$, with $i=1,2,3$. Assume a symmetric payoff function such that:

$$
u_{i}(x)=x_{i}+\sum_{j \in-i} x_{j}, i=1,2,3
$$

We know that $\bar{x} \in X$ is a BZE if and only if $\max _{y_{-i} \in X_{-i}} u_{i}\left(\bar{x}_{i}, y_{-i}\right)=u_{i}(\bar{x})$, for any $i=1,2,3$. We deduce that $\bar{x}=(1,1,1)$ is the unique pure strategy BZE of this game with $u_{i}(\bar{x})=3$, for all $i=1,2,3$. This BZE is also a NE as $\underline{x}=(1,1,1)$ with $u_{i}(\underline{x})=3$, for all $i=1,2,3$, this is a BNE.

EXAMPle 2.4 (Climate coalitional game) Considering now the climate coalition game defined in example 2.1 and assuming that $R=\{\{1\},\{2\},\{3\},\{4\}\}$, the unique coalitional Berge equilibrium is given by

$$
\bar{e}_{i}=\frac{\alpha_{i}^{2}}{4 \beta_{i}^{2}}, i=1, \ldots, 4 .
$$

This equilibrium is also the unique Nash equilibrium of the coalitional game, this is a BNE

We obtain the following proposition that can be extended to games in individualistic form.

Proposition 2.1 Consider the game (2.1) and let $R=\left\{R_{i}\right\}_{i \in M} \subset \Im$ be a partition of I (coalition structure) and $S=\left\{S_{i}\right\}_{i \in M}$ be a set of subsets of $I$. If each $m \in M$, we have $r_{m} \in S_{m}$, and thus each CBE is also a NE.

Proof. By the definition of CBE (see Definition 2.1).

BNE is by definition immune from unilateral deviation.

\section{Existence Results}

In order to establish general existence results for BVE (Definition 2.3), BNE (Definition 2.4) and $\mathrm{CBE}^{3}$ (Definition 2.1), let us first restate the generalizations of Baye et al. (1993)'s definitions of transfer continuity and transfer quasiconcavity to a function defined in the product of different sets.

Definition 3.1 ( $g$-Diagonal Transfer Continuity). Consider $X, Y$ two topological spaces, $f$ : $X \times Y \rightarrow \mathbb{R}$ and $g: X \rightarrow Y$ two functions. The function $f$ is said to be $g$-diagonal transfer continuous if for every $(x, y) \in X \times Y, f(x, y)>f(x, g(x))$ implies that there exists $y^{\prime} \in Y$ and a neighborhood $\mathcal{V}_{x}$ of $x$ such that: $f\left(z, y^{\prime}\right)>f(z, g(z))$, for each $z \in \mathcal{V}_{x}$.

\footnotetext{
${ }^{3} \mathrm{BZE}$ (Definition 2.2) is a special case of CBE.
} 
The $g$-diagonal transfer continuity says that if a point $x$ in $X$ is dominated by another point $y$ in $Y$ (in the sense $f(x, y)>f(x, g(x))$ ), then there is an open set of points containing $x$, that all can be strictly dominated by a single point $y^{\prime}$. In other words, $g$-diagonal transfer continuity refers to the fact that $y$ may be transferred to some $y^{\prime}$ in order for the inequality to hold for all points in a neighborhood of $x$.

Proposition 3.1 Any one of the following conditions is sufficient for $f$ to be g-diagonal transfer continuous:

a) $f$ and $g$ are continuous;

b) $f(x, y)$ is transfer lower semicontinuous in $x$ and $f(x, g(x))$ is upper semicontinuous in $x .^{4}$

Proof. Item a) is a straightforward consequence of the definition of the continuity of $f$ and $g$. In order to prove item b), assume that $f(x, y)>f(x, g(x))$. For some $\epsilon>0$, we have $f(x, y)>f(x, g(x))+2 \epsilon$. Since $f(x, y)$ is transfer lower semicontinuous in $x$ and $f(x, g(x))$ is upper semicontinuous in $x$, there exists for $\epsilon>0$, a neighborhood $\mathcal{V}_{x}$ of $x$ and a point $\tilde{y} \in Y$ such that for each $z \in \mathcal{V}_{x}$, we have

$$
\left\{\begin{array}{l}
f(z, \tilde{y}) \geq f(x, y)-\epsilon \\
f(x, g(x)) \geq f(z, g(z))-\epsilon .
\end{array}\right.
$$

We deduce that for each $z \in \mathcal{V}_{x}, f(z, \tilde{y}) \geq f(x, y)-\epsilon>f(x, g(x))+\epsilon \geq f(z, g(z))$.

DeFinition 3.2 (g-Diagonal Transfer Quasiconcavity) Let $X$ be a nonempty convex subset of a vector space $E, Y$ be a nonempty set and $g: X \rightarrow Y$ be a function. A function $f: X \times Y \rightarrow \mathbb{R}$ is said to be $g$-diagonal transfer quasiconcave in $y$ if, for any finite subset $\left\{y^{1}, \ldots, y^{p}\right\} \subset Y$, there exists a corresponding finite subset $\left\{x^{1}, \ldots, x^{p}\right\} \subset X$ such that for any subset $J \subset\{1,2, \ldots, p\}$ and any $x$ in the convex hull of the set $\left\{x^{h}: h \in J\right\}$, we have $\min _{h \in J} f\left(x, y^{h}\right) \leq f(x, g(x))$.

The $g$-diagonal transfer quasiconcavity in $y$ says that, given any finite set $\left\{y^{1}, \ldots, y^{p}\right\} \subset$ $Y$, there exists a corresponding finite subset $\left\{x^{1}, \ldots, x^{p}\right\} \subset X$ such that for any subset $\left\{x^{j_{h}}, \ldots, x^{j_{k}}\right\} \subset\left\{x^{1}, \ldots, x^{p}\right\}$, and for any $x$ in the convex hull of the set $\left\{x^{j_{1}}, \ldots, x^{j_{k}}\right\},(x, g(x))$ is never dominated by all deviations in $\left\{\left(x, y^{j_{1}}\right), \ldots,\left(x, y^{j_{k}}\right)\right\}$.

Proposition 3.2 Suppose that $X=Y$. Any one of the following conditions is sufficient for $f$ to be $g$-diagonal transfer quasiconcave:

\footnotetext{
${ }^{4}$ A function $f(x, y)$ is said to be transfer lower semicontinuous in $x$, if for each $\epsilon>0$ there exists a neighborhood $\mathcal{V}_{x}$ of $x$ and a point $\tilde{y} \in Y$ such that $f(z, \tilde{y}) \geq f(x, y)-\epsilon$, for each $z \in \mathcal{V}_{x}$. A function $f(x, g(x))$ is said to be upper semicontinuous in $x$, if for each $\epsilon>0$ there exists a neighborhood $\mathcal{V}_{x}$ of $x$ such that $f(x, g(x)) \geq f(z, g(z))-\epsilon$, for each $z \in \mathcal{V}_{x}$.
} 
a) $y \mapsto f(x, y)$ is concave or quasiconcave;

b) $y \mapsto f(x, y)$ is g-diagonally quasiconcave. ${ }^{5}$

Proof. Item a) is a straightforward consequence of the definition of concavity and/or quasiconcavity. In order to prove item $\mathbf{b}$ ), fix any finite subset $\left\{y^{1}, \ldots, y^{p}\right\} \subset Y$. There exists a corresponding finite subset $\left\{x^{1}, \ldots, x^{p}\right\} \subset X$ such that $x^{h}=y^{h}$, for $h=1, \ldots, p$. Since $f(x, y)$ is $g$ diagonally quasiconcave in $y$, then for any subset $J \subset\{1,2, \ldots, p\}$ and any $x \in \operatorname{co}\left\{x^{h}: h \in J\right\}$, we have $\min _{h \in J} f\left(x, y^{h}\right) \leq f(x, g(x))$.

The following generalization of the Ky Fan inequality (Fan 1972) is established in Nessah and Tazdaï (2013). It generalizes most minimax theorems proposed in the literature, e.g. Tian and Zhou (1993), Nessah and Larbani (2004), Nessah et al. (2009). ${ }^{6}$

LEMMA 3.1 (Nessah and Tazdaït (2013)) Let $X$ be a nonempty, compact and convex subset of a locally convex Hausdorff space $E, Y$ be a nonempty subset of a topological space $F$. Let $g$ be a function defined from $X$ into $Y$ and $\Omega$ be a real valued g-diagonal transfer continuous function defined on $X \times Y$. Then $\Omega$ is $g$-diagonal transfer quasiconcave in $y$ if and only if there exists $\bar{x} \in X$ such that $\sup _{y \in Y} \Omega(\bar{x}, y)=\Omega(\bar{x}, g(\bar{x}))$.

\subsection{Berge-Vaisman equilibrium}

We now establish the existence of BVE in the game (2.1) by using Lemma 3.1. Let us consider the set $A$ of individually rational strategy profiles of the game (2.1).

$$
A=\left\{\bar{x} \in X \text { such that } \alpha_{i}=\max _{x_{i} \in X_{i} y_{-i} \in X_{-i}} u_{i}\left(x_{i}, y_{-i}\right) \leq u_{i}(\bar{x}), \forall i \in I\right\}
$$

LEMма 3.2 Suppose that the following conditions are satisfied:

1) for all $i \in I$, the set $X_{i}$ is non empty, convex and compact in the Hausdorff locally convex space $E_{i}$,

2) for all $i \in I$, the function $u_{i}$ is continuous and quasiconcave on $X$.

Then, the set A defined in (3.1) is nonempty, convex and compact.

Let us define two functions we denote $g$ and $\Gamma$ :

$$
g: A \rightarrow \widehat{X}
$$

\footnotetext{
${ }^{5} \mathrm{~A}$ function $f(x, y)$ is said to be $g$-diagonally quasiconcave in $y$ if for every finite subset $\left\{y^{1}, \ldots, y^{p}\right\} \subset X$ and any $y \in\left\{y^{1}, \ldots, y^{p}\right\}$, we have $\min _{h=1, \ldots, p} f\left(y, y^{h}\right) \leq f(y, g(y))$.

${ }^{6}$ For more details on these generalizations, see Nessah and Tian (2013).
} 
defined by $x \mapsto g(x)=\left(x_{-1}, \ldots, x_{-n}\right)$, and

$$
\Gamma: A \times \widehat{X} \rightarrow \mathbb{R}
$$

defined by $(x, \widehat{y}) \mapsto \Gamma(x, \widehat{y})=\sum_{i \in I} u_{i}\left(x_{i}, y_{-i}\right) \quad$ where $\widehat{y}=\left(y_{-1}, \ldots, y_{-n}\right) \in \widehat{X}=\prod_{i \in I} X_{-i}$, and $X_{-i}=\prod_{j \in-i} X_{j}, \forall i \in I$

REMARK 3.1 For all $x \in A$, we have

$$
\sup _{\widehat{y} \in \widehat{X}} \Gamma(x, \widehat{y}) \geq \Gamma(x, g(x))
$$

The next Lemma establishes the relation between BVE of the game (2.1) and the functions $\Gamma$ and $g$.

LEMMA 3.3 The following two assertions are equivalent.

1) $\bar{x}$ is a BVE of the game (2.1).

2) $\bar{x} \in A$ and $\sup _{\widehat{X}} \Gamma(\bar{x}, \widehat{y})=\Gamma(\bar{x}, g(\bar{x}))$

$$
\widehat{y} \in \widehat{X}
$$

REMARK 3.2 Lemma 3.3 transforms the problem of finding BVE of the game (2.1) into finding a strategy profile $\bar{x} \in A$ satisfying $\sup \Gamma(\bar{x}, \widehat{y})=\Gamma(\bar{x}, g(\bar{x}))$.

$$
\widehat{y} \in \widehat{X}
$$

Let

$$
\alpha=\inf _{x \in X}\left[\sup _{\widehat{y} \in \widehat{X}} \Gamma(x, \widehat{y})-\Gamma(x, g(x))\right] .
$$

From Remark 3.1 and Lemma 3.3, we establish the following proposition for games with a finite number of players.

Proposition 3.3 Suppose that $u_{j}$ is continuous on $X$ and the set $X_{j}$ is compact for each $j \in I$. The game (2.1) admits at least one BVE if and only if $\alpha=0$.

A procedure to compute BVE of a continuous and compact game is immediately deduced from this proposition. It suffices to prove that $\alpha=0$, and show that the corresponding strategy profile is also a maximin profile. The following example illustrates this computation method. ${ }^{7}$

EXAMPLE 3.1 Suppose a three player game such that $I=\{1,2,3\}, X_{i}=[-1,1], i=1,2,3$, and $x=\left(x_{1}, x_{2}, x_{3}\right)$. Players' utility functions are such that:

\footnotetext{
${ }^{7}$ Note that efficient algorithmic computation of Berge equilibrium and refinements is a hard problem. Preliminary results of PPAD completeness for computing Berge equilibrium and refinements in two-player games are provided in the appendix. We thank an anonymous referee for having raised this important issue.
} 


$$
\begin{gathered}
u_{1}(x)=x_{1}+x_{2}-x_{3}^{2}, \\
u_{2}(x)=x_{2}-x_{3}^{2}, \\
u_{3}(x)=x_{1}+x_{2}^{2}\left(x_{3}+1\right) .
\end{gathered}
$$

Notice first that the game is continuous and compact. Notice second that $\max _{y_{-1}} u_{1}\left(x_{1}, y_{-1}\right)=$ $u_{1}\left(x_{1}, 1,0\right), \max _{y_{-2}} u_{2}\left(x_{2}, y_{-2}\right)=u_{2}\left(y_{1}, x_{2}, 0\right)$ and $\max _{y_{-3}} u_{3}\left(x_{3}, y_{-3}\right)=u_{3}\left(1, \pm 1, x_{3}\right)$. We have $\sum_{i=1}^{3} \max _{y_{-i}}\left[u_{i}\left(x_{i}, y_{-i}\right)-u_{i}(x)\right]=-x_{1}-x_{2}+x_{3}+2 x_{3}^{2}-x_{2}^{2}-x_{3} x_{2}^{2}+3$.

Notice finally that $\alpha=\inf _{x \in X}\left[-x_{1}-x_{2}+x_{3}+2 x_{3}^{2}-x_{2}^{2}-x_{3} x_{2}^{2}+3\right]=0$ and this minimum is reached in $\bar{x}=(1,1,0)$. Since $\alpha_{1}=0, \alpha_{2}=0$ and $\alpha_{3}=-1$, we have $\bar{x} \in A$. According to Proposition $3.3, \bar{x}=(1,1,0)$ is a BVE of this game.

From Lemmas $(3.1,3.2,3.3)$ and Proposition 3.1, we derive the following existence result which constitutes the first existence result for BVE that has been provided in the literature to date.

THEOREM 3.1 Assume that the sets $X_{i}, i \in I$, are non empty compact and convex subsets of locally convex Hausdorff spaces, and the function $u_{i}$ is continuous and quasiconcave on $X, i \in I$. Then, the game (2.1) admits at least one BVE (Definition 2.3) if and only if $\Gamma$ is $g$-diagonal transfer quasiconcave in $\widehat{y}$.

Proof. The assumptions of Theorem 3.1 imply that conditions of Lemma 3.2 are satisfied. It follows that the set $A^{\prime}$ is nonempty, convex and compact. By Proposition 3.1, the function $\Gamma$ is $g$-diagonal transfer continuous. Then, by Lemma 3.1 and Lemma 3.3, the game (2.1) admits at least one BVE if and only if there exists $\bar{x} \in A$ such that $\sup \Gamma(\bar{x}, \widehat{y})=\Gamma(\bar{x}, g(\bar{x}))$.

$$
\widehat{y} \in \widehat{X}
$$

Let us now relax the continuity of $u_{i}$ and the quasiconcavity of $u_{i}$ in $x$. Consider $A^{\prime}$ a modified set of $A$ :

$$
A^{\prime}=\left\{\bar{x} \in X \text { such that } \alpha_{i}=\sup _{x_{i} \in X_{i}{ }_{x_{-i} \in X_{-i}}} u_{i}\left(x_{i}, x_{-i}\right) \leq \inf _{x_{-i} \in X_{-i}} u_{i}\left(\bar{x}_{i}, x_{-i}\right), \forall i \in I\right\} .
$$

We have $A^{\prime} \subset A$. Indeed, if $\bar{x} \in A^{\prime}$, then for each $i \in I, \alpha_{i} \leq \inf _{x_{-i} \in X_{-i}} u_{i}\left(\bar{x}_{i}, x_{-i}\right) \leq u_{i}(\bar{x})$. The converse is not true in general.

LEMMA 3.4 The set $A^{\prime}$ is nonempty, convex and compact if the three following conditions are satisfied:

1) for all $i \in I$, the set $X_{i}$ is non empty, convex and compact in the Hausdorff locally convex space $E_{i}$,

2) for all $i \in I$, the function $\inf _{x_{-i} \in X_{-i}} u_{i}\left(., x_{-i}\right)$ is upper semicontinuous on $X_{i}$, i.e. for each $x_{i}, \epsilon>0$, there exists a neighborhood $\mathcal{V}_{x_{i}}$ of $x_{i}$ such that for each $z_{i} \in \mathcal{V}_{x_{i}}$, $\inf _{x_{-i} \in X_{-i}} u_{i}\left(z_{i}, x_{-i}\right) \leq \inf _{x_{-i} \in X_{-i}} u_{i}\left(x_{i}, x_{-i}\right)+\epsilon$. 
3) for all $i \in I$, the function $\inf _{x_{-i} \in X_{-i}} u_{i}\left(., x_{-i}\right)$ is quasiconcave on $X_{i}$.

Denoting $g^{\prime}=\left.g\right|_{A^{\prime}}$ the restriction of $g$ on $A^{\prime}$ and $\Gamma^{\prime}=\left.\Gamma\right|_{A^{\prime} \times \widehat{X}}$ the restriction of $\Gamma$ on $A^{\prime} \times$ $\widehat{X}$, a sufficient existence condition for BVE follows. This existence result is more general than Theorem 3.1 as we relaxed continuity and quasconcavity conditions.

THEOREM 3.2 Assume that the sets $X_{i}, i \in I$, are non empty compact and convex subsets of locally convex Hausdorff spaces, and the function $\inf _{x_{-i} \in X_{-i}} u_{i}\left(., x_{-i}\right)$ is upper semicontinuous and quasiconcave on $X_{i}, i \in I$. If $\Gamma^{\prime}$ is $g^{\prime}$-diagonal transfer continuous and $g^{\prime}$-diagonal transfer quasiconcave in $\widehat{y}$ then the game (2.1) admits at least one BVE (Definition 2.3).

Proof. The assumptions of Theorem 3.1 imply that conditions of Lemma 3.4 are satisfied. It follows that the set $A^{\prime}$ is nonempty, convex and compact. By Lemma 3.1, there exists $\bar{x} \in A^{\prime}$ such that $\sup \Gamma^{\prime}(\bar{x}, \widehat{y})=\Gamma^{\prime}\left(\bar{x}, g^{\prime}(\bar{x})\right)$. Since $A^{\prime} \subset A$, we deduce that $\bar{x}$ is a BVE of game (2.1). $\widehat{y} \in \widehat{X}$

In order to illustrate the generality of this result, let us consider a game example that is neither continuous nor quasiconcave.

EXAMPLE 3.2 (Discontinuous game) Let $I=\{1,2,3\}, X_{i}=[0,1]$, with $i=1,2,3$, and $x=$ $\left(x_{1}, x_{2}, x_{3}\right)$. Further assume that:

$$
\begin{gathered}
u_{1}(x)=\left\{\begin{array}{ll}
x_{1}+2 & \text { if } x_{2} \geq x_{3} \\
x_{1}+x_{2}+x_{3} & \text { otherwise }
\end{array}, u_{2}(x)= \begin{cases}x_{2}+2 & \text { if } x_{1} \geq x_{3} \\
x_{1}+x_{2}+x_{3} & \text { otherwise }\end{cases} \right. \\
u_{3}(x)= \begin{cases}x_{3}+2 & \text { if } x_{1} \geq x_{2} \\
x_{1}+x_{2}+x_{3} & \text { otherwise }\end{cases}
\end{gathered}
$$

This game is neither continuous nor quasiconcave on $X$ but for each $i=1,2,3$, $\inf _{x_{-i} \in X_{-i}} u_{i}\left(x_{i}, x_{-i}\right)=x_{i}$, meaning that the function $\inf _{x_{-i} \in X_{-i}} u_{i}\left(., x_{-i}\right)$ is upper semicontinuous and quasiconcave on $X_{i}$. Notice that $\Gamma^{\prime}$ is $g^{\prime}$-diagonal transfer continuous. Indeed, let $x$ not be a BVE. Then, there exists $\widehat{y} \in \widehat{X}$ (defined by $\widehat{y}_{j}=1$, for each $j$ ) and a neighborhood $\mathcal{V}_{x}$ of $x$ such that for each $z \in \mathcal{V}_{x}, \Gamma^{\prime}(z, \widehat{y})>\Gamma^{\prime}\left(z, g^{\prime}(z)\right)$. $\Gamma^{\prime}$ is also $g^{\prime}$-diagonal transfer quasiconcave in $\widehat{y}$. Indeed, for any finite subset $\left\{\widehat{y}^{1}, \ldots, \widehat{y}^{p}\right\} \subset \widehat{X}$, there exists a corresponding finite subset $\left\{x^{1}, \ldots, x^{p}\right\}=\{(1,1,1)\} \subset A^{\prime}$ such that for any subset $J \subset\{1,2, \ldots, p\}$ and any $x \in \operatorname{co}\left\{x^{h}: h \in J\right\}, \min _{h \in J} \Gamma^{\prime}\left(x, \widehat{y}^{h}\right) \leq \Gamma^{\prime}\left(x, g^{\prime}(x)\right)$. By Theorem 3.2, this game admits a BVE.

\subsection{Berge-Nash Equilibrium}

We now establish conditions for existence of BNE by using Lemma 3.1. Again, let us consider two functions we denote $\widetilde{g}$ and $\widetilde{\Gamma}$.

$$
\widetilde{g}: X \rightarrow \widehat{X} \times X
$$


defined by $x \mapsto \widetilde{g}(x)=\left(\left(x_{-1}, \ldots, x_{-n}\right), x\right)$ and

$$
\widetilde{\Gamma}: X \times(\widehat{X} \times X) \rightarrow \mathbb{R}
$$

defined by $(x,(\widehat{y}, z)) \mapsto \widetilde{\Gamma}(x,(\widehat{y}, z))=\Gamma(x, \widehat{y})+\sum_{i \in I} u_{i}\left(x_{-i}, z_{i}\right)=\sum_{i \in I}\left[u_{i}\left(x_{i}, y_{-i}\right)+u_{i}\left(x_{-i}, z_{i}\right)\right]$.

REMARK 3.3 By definition, for all $x \in X$, we have

$$
\sup _{(\widehat{y}, z) \in \widehat{X} \times X} \widetilde{\Gamma}(x,(\widehat{y}, z)) \geq \widetilde{\Gamma}(x, \widetilde{g}(x)) .
$$

The following Lemma establishes the relation between BNE of the game (2.1) and the functions $\widetilde{\Gamma}$ and $\widetilde{g}$.

\section{LEMMA 3.5 The following two assertions are equivalent:}

$$
\text { 1) } \sup _{(\widehat{y}, z) \in \widehat{X} \times X} \widetilde{\Gamma}(\bar{x},(\widehat{y}, z))=\widetilde{\Gamma}(\bar{x}, \widetilde{g}(\bar{x})) \text {. }
$$

2) $\bar{x}$ is a BNE of the game (2.1).

Note that in Lemma 3.5, we deliberately omitted the individual rationality condition $\bar{x} \in A$. Indeed it is a well known result that a NE is always individually rational.

Let

$$
\beta=\inf _{x \in X}\left[\sup _{(\widehat{y}, z) \in \widehat{X} \times X} \widetilde{\Gamma}(x,(\widehat{y}, z))-\widetilde{\Gamma}(x, \widetilde{g}(x))\right] .
$$

We deduce from Remark 3.3 and Lemma 3.5, the following proposition.

Proposition 3.4 Suppose that the function $u_{i}$ is continuous on $X$ and the set $X_{i}$ is compact, for each $i \in I$. Then, the game (2.1) has at least one BNE if and only if $\beta=0$.

Notice that the existence and the computation of BNE in continuous and compact games is immediately deduced from this proposition. To illustrate it, consider again the game described in Example 3.1.

EXAMPLE 3.3 Let $I=\{1,2,3\}, X_{i}=[-1,1], i=1,2,3$, and $x=\left(x_{1}, x_{2}, x_{3}\right)$. Players' utility functions are such that:

$$
\begin{gathered}
u_{1}(x)=x_{1}+x_{2}-x_{3}^{2}, \\
u_{2}(x)=x_{2}-x_{3}^{2}, \\
u_{3}(x)=x_{1}+x_{2}^{2}\left(x_{3}+1\right) .
\end{gathered}
$$


Notice first that the game is continuous and compact. According to Proposition 3.4, there is at least one BNE if and only if $\beta=0$. By Example 3.1, we have

$$
\sup _{\widehat{y} \in \widehat{X}} \Gamma(x, \widehat{y})-\Gamma(x, g(x))=-x_{1}-x_{2}+x_{3}+2 x_{3}^{2}-x_{2}^{2}-x_{3} x_{2}^{2}+3,
$$

and

$$
\sum_{i=1}^{3} \max _{z_{i}}\left[u_{i}\left(z_{i}, x_{-i}\right)-u_{i}(x)\right]=2-x_{1}-x_{2}+\left(1-x_{3}\right) x_{2}^{2} .
$$

We deduce that $\beta=\inf _{x \in X}\left[5-2 x_{1}-2 x_{2}+x_{3}+2 x_{3}^{2}-2 x_{3} x_{2}^{2}\right]=\frac{7}{8}$ and by Proposition 3.4, we concude that the game has no BNE.

As for BVE, let us now relax continuity and quasiconcavity conditions. We have the following existence Theorem.

THEOREM 3.3 Suppose that the sets $X_{i}, i \in I$, are nonempty, compact and convex subsets of Hausdorff locally convex vector spaces and the function $\widetilde{\Gamma}$ is $\widetilde{g}$-diagonal transfer continuous. Then the game (2.1) has at least one BNE (Definition 2.4) if and only if the function $\widetilde{\Gamma}$ is $\widetilde{g}$-diagonal transfer quasiconcave in $(\widehat{y}, z)$.

Proof. The conditions of Theorem 3.3 imply that all conditions of Lemma 3.1 are satisfied. It follows that by Lemma 3.5, the game (2.1) has at least one Berge-Nash equilibrium (Definition 2.4) if and only if the function $\widetilde{\Gamma}$ is $\widetilde{g}$-diagonal transfer quasiconcave in $(\widehat{y}, z)$.

In order to illustrate the generality of this existence result, consider again the discontinuous game described in Example 3.2. In this example, utility functions are not lower semicontinuous and the existence results provided in Larbani and Nessah (2008) do not apply.

EXAMPle 3.4 (Discontinuous game) Let $I=\{1,2,3\}, X_{i}=[0,1], i=1,2,3$ and $x=$ $\left(x_{1}, x_{2}, x_{3}\right)$. Players' utility functions are:

$$
\begin{aligned}
& u_{1}(x)=\left\{\begin{array}{ll}
x_{1}+2 & \text { if } x_{2} \geq x_{3} \\
x_{1}+x_{2}+x_{3} & \text { otherwise }
\end{array}, u_{2}(x)= \begin{cases}x_{2}+2 & \text { if } x_{1} \geq x_{3} \\
x_{1}+x_{2}+x_{3} & \text { otherwise }\end{cases} \right. \\
& u_{3}(x)= \begin{cases}x_{3}+2 & \text { if } x_{1} \geq x_{2} \\
x_{1}+x_{2}+x_{3} & \text { otherwise }\end{cases}
\end{aligned}
$$

Notice first, that the functions $x_{-i} \mapsto u_{i}\left(x_{i}, x_{-i}\right)$ are not lower semicontinuous. Indeed, let $x_{1} \in[0,1]$ and $\left(x_{2}, x_{3}\right)=(0,0)$. There exists $\epsilon=1>0$ such that for each neighborhood $\mathcal{V}$ of $(0,0)$, there exists $\left(x_{2}^{\prime}, x_{3}^{\prime}\right) \in \mathcal{V}$ (with $x_{2}^{\prime}<x_{3}^{\prime}<\frac{\epsilon}{2}$ ) so as $u_{1}\left(x_{1}, x_{2}^{\prime}, x_{3}^{\prime}\right)=x_{1}+x_{2}^{\prime}+x_{3}^{\prime}<$ $x_{1}+2-\epsilon=u_{1}\left(x_{1}, 0,0\right)-\epsilon$. We deduce that Theorems $(4.2,4.3,4.4)$ provided in Larbani and Nessah (2008) cannot be applied. 
Notice second that $\widetilde{\Gamma}$ is $\widetilde{g}$-diagonal transfer continuous. Indeed, let $x$ not be a BNE. Then, there exists $(\widehat{y}, t) \in \widehat{X} \times X$ defined by $\widehat{y}_{j}=1$, for each $j$ and $t=(1,1,1)$ and a neighborhood $\mathcal{V}_{x}$ of $x$ such that for each $z \in \mathcal{V}_{x}, \widetilde{\Gamma}(z,(\widehat{y}, t))>\widetilde{\Gamma}(z, \widetilde{g}(z))$. $\widetilde{\Gamma}$ is also $\widetilde{g}$-diagonal transfer quasiconcave in $(\widehat{y}, z)$. Indeed, for any finite subset $\left\{\left(\widehat{y}^{1}, z^{1}\right), \ldots,\left(\widehat{y}^{p}, z^{p}\right)\right\} \subset \widehat{X}$, there exists a corresponding finite subset $\left\{x^{1}, \ldots, x^{p}\right\}=\{(a, a, a)\}$ (with $a=\max _{i, j, h=1, \ldots, p}\left\{\widehat{y}_{j}^{h}, z_{i}^{h}\right\}$ ) such that for any subset $J \subset\{1,2, \ldots, p\}$ and any $x \in \operatorname{co}\left\{x^{h}: \quad h \in J\right\}$, we have $\min _{h \in J} \widetilde{\Gamma}\left(x,\left(\widehat{y}^{h}, z^{h}\right)\right) \leq \widetilde{\Gamma}(x, \widetilde{g}(x))$. By Theorem 3.3, this game admits a BNE.

\subsection{Coalitional Berge Equilibrium}

We now establish conditions for existence of CBE (Definition 2.1) in the game (2.1) from which we deduce conditions for existence of BZE (Definition 2.2).

Let $R=\left\{R_{i}\right\}_{i \in M} \subset \Im$ be a partition of $I$ and $S=\left\{S_{i}\right\}_{i \in M}$ be a set of subsets of $I$.

Again, consider two functions we now denote $h$ and $F$ :

$$
h: X \rightarrow \widetilde{X}
$$

defined by $x \mapsto h(x)=(\overbrace{\left(x_{S_{m}}, \ldots, x_{S_{m}}\right)}^{r_{m} \text {-times }}, m \in M)$ and

$$
F: X \times \tilde{X} \rightarrow \mathbb{R}
$$

defined by $(x, \widetilde{y}) \mapsto F(x, \widetilde{y})=\sum_{m \in M} \sum_{j \in R_{m}}\left\{u_{j}\left(x_{-S_{m}}, y_{S_{m}}\right)-u_{j}(x)\right\}$, where $\widetilde{X}=\prod_{m \in M} \prod_{j \in R_{m}} X_{S_{m}}^{j}$ and $X_{S_{m}}^{j}=X_{S_{m}}, \forall j \in R_{m}$.

Lemma 3.6 establishes the relation between CBE of the game (2.1) and the functions $F$ and $h$.

LЕМмA 3.6 The following two propositions are equivalent:

1) $\sup _{\widetilde{y} \in \widetilde{X}} F(\bar{x}, \widetilde{y})=0$.

2) $\bar{x}$ is a CBE of the game (2.1).

Let

$$
\gamma=\inf _{x \in X}\left[\sup _{\widetilde{y} \in \widetilde{X}} F(x, \widetilde{y})\right] .
$$

By Lemma 3.6, we deduce the following proposition.

Proposition 3.5 Suppose that the function $u_{i}$ is continuous on $X$ and the set $X_{i}$ is compact, for each $i \in I$. Then, the game (2.1) has at least one CBE (Definition 2.1) if and only if $\gamma=0$. 
As for BVE and BNE, existence and computation of CBE in a game that is compact and continuous is immediately deduced from this proposition. Computation of BZE is also straightforward as BZE is a special case of CBE when $M=I, R_{i}=\{i\}, i \in I, S_{i}=-i$, and $i \in I$. Let us illustrate this result with an example.

EXAMPLE 3.5 Let $I=\{1,2,3\}, X_{1}=X_{2}=X_{3}=[0,1]$ and

$$
\begin{gathered}
u_{1}(x)=x_{1}+x_{2}+x_{3}, \\
u_{2}(x)=-x_{1}+x_{2}-x_{3}, \\
u_{3}(x)=x_{1}-x_{2}+x_{3} .
\end{gathered}
$$

It can be easily seen that this game is compact and continuous.

Consider first the case where $M=I, R_{i}=\{i\}, i \in I$ and $S_{i}=-i, i \in I$. That is, the case where there is no coalition but agents playing individually. We can show that the game admits no BZE. Indeed, $\widetilde{X}$ becomes $\widetilde{X}=X_{-1} \times X_{-2} \times X_{-3}$ and

$$
F(x, \widetilde{y})=\left[u_{1}\left(x_{1}, y_{-1}\right)-u_{1}(x)\right]+\left[u_{2}\left(x_{2}, y_{-2}\right)-u_{2}(x)\right]+\left[u_{3}\left(x_{3}, y_{-3}\right)-u_{3}(x)\right],
$$

where $x \in X$ and $\widetilde{y}=\left(y_{-1}, y_{-2}, y_{-3}\right) \in \widetilde{X}$.

Let $x \in X$, we have:

$$
\begin{gathered}
\max _{y_{-1}} u_{1}\left(x_{1}, y_{-1}\right)=u_{1}\left(x_{1}, 1,1\right)=x_{1}+2, \\
\max _{y_{-2}} u_{2}\left(x_{2}, y_{-2}\right)=u_{2}\left(0, x_{2}, 0\right)=x_{2}, \\
\max _{y_{-3}} u_{3}\left(x_{3}, y_{-3}\right)=u_{3}\left(1,0, x_{3}\right)=x_{3}+1 .
\end{gathered}
$$

Therefore $\sum_{i=1}^{3} \max _{y_{-i}} u_{i}\left(x_{i}, y_{-i}\right)=x_{1}+x_{2}+x_{3}+3$. Since $\gamma=\inf _{x \in X}\left[\sup _{\widetilde{y} \in \widetilde{X}} F(x, \widetilde{y})\right]$. Then, we have

$$
\gamma=\inf _{x \in X}\left[\left(x_{1}+2-x_{1}-x_{2}-x_{3}\right)+\left(x_{2}+x_{1}-x_{2}+x_{3}\right)+\left(x_{3}+1-x_{1}+x_{2}-x_{3}\right)\right]=3
$$

and by Proposition 3.5, we conclude that the game admits no BZE.

Consider second a coalitional case such that $R=\{i\}_{i \in I}$ and $S=\left\{S_{i}\right\}_{i \in I}$ defined by $S_{1}=$ $\{1,2\}, S_{2}=\{2,3\}$ and $S_{3}=\{1\}$. The game admits an equilibrium point for the set $R$ relative to the set $S$. Indeed, $\widetilde{X}$ becomes $\widetilde{X}=X_{-3} \times X_{-1} \times X_{1}$ and

$$
F(x, \widetilde{y})=\left[u_{1}\left(y_{1}^{1}, y_{2}^{1}, x_{3}\right)-u_{1}(x)\right]+\left[u_{2}\left(x_{1}, y_{2}^{2}, y_{3}^{2}\right)-u_{2}(x)\right]+\left[u_{3}\left(y_{1}^{3}, x_{2}, x_{3}\right)-u_{3}(x)\right],
$$

with $x \in X$ and $\widetilde{y}=\left(\left(y_{1}^{1}, y_{2}^{1}\right),\left(y_{2}^{2}, y_{3}^{2}\right), y_{1}^{3}\right) \in \widetilde{X}$.

Let $x \in X$, we have 


$$
\begin{gathered}
\max _{y_{1}^{1}, y_{2}^{1}} u_{1}\left(y_{1}^{1}, y_{2}^{1}, x_{3}\right)=u_{1}\left(1,1, x_{3}\right)=x_{3}+2, \\
\max _{y_{2}^{2}, y_{3}^{2}} u_{2}\left(x_{1}, y_{2}^{2}, y_{3}^{2}\right)=u_{2}\left(x_{1}, 1,0\right)=-x_{1}+1, \\
\max _{y_{1}^{3}} u_{3}\left(y_{1}^{3}, x_{2}, x_{3}\right)=u_{3}\left(1, x_{2}, x_{3}\right)=-x_{2}+x_{3}+1 .
\end{gathered}
$$

Notice that $\sum_{i=1}^{3} \max _{y_{S_{i}}} u_{i}\left(x_{-S_{i}}, y_{S_{i}}\right)=-x_{1}-x_{2}+2 x_{3}+4$ and $\sum_{i=1}^{3} u_{i}(x)=x_{1}+x_{2}+x_{3}$.

As $\gamma=\inf _{x \in X}\left[\sup _{\widetilde{y} \in \widetilde{X}} F(x, \widetilde{y})\right]$, we obtain: $\gamma=\inf _{x \in X}\left[4-2 x_{1}-2 x_{2}+x_{3}\right]=0$. By Proposition 3.5, $\bar{x}=(1,1,0)$ is a CBE of the game.

We now relax the continuity and quasiconcavity conditions and find sufficiency conditions for the existence of CBE. As for Theorem 3.1, we have the following existence result.

THEOREM 3.4 Suppose that the sets $X_{i}, i \in I$, are nonempty, compact and convex subsets of Hausdorff locally convex vector spaces, and the function $F$ is h-diagonal transfer continuous. Then, the game (2.1) has at least one CBE (Definition 2.1) if and only if $F$ is h-diagonal transfer quasiconcave in $\widetilde{y}$.

ProOF. The proof of this theorem is similar to that of Theorem 3.1.

To illustrate the generality of this theorem, consider the discontinuous game described in Example 3.2. Again, because in this example $u_{i}($.$) is not continuous and u_{i}\left(x_{i},.\right)$ is not lower semicontinuous, the existence results provided in the literature do not apply.

EXAMPLE 3.6 (Discontinuous game) We have: $I=\{1,2,3\}, X_{i}=[0,1], i=1,2,3$, and $x=\left(x_{1}, x_{2}, x_{3}\right)$. Players' utility functions are:

$$
\begin{gathered}
u_{1}(x)=\left\{\begin{array}{ll}
x_{1}+2 & \text { if } x_{2} \geq x_{3} \\
x_{1}+x_{2}+x_{3} & \text { otherwise }
\end{array}, u_{2}(x)= \begin{cases}x_{2}+2 & \text { if } x_{1} \geq x_{3} \\
x_{1}+x_{2}+x_{3} & \text { otherwise }\end{cases} \right. \\
u_{3}(x)= \begin{cases}x_{3}+2 & \text { if } x_{1} \geq x_{2} \\
x_{1}+x_{2}+x_{3} & \text { otherwise }\end{cases}
\end{gathered}
$$

Let $R_{m}=S_{m}$ and assume that $m \in R_{m}$, for each $m=1,2,3$. Notice that $F$ is $h$-diagonal transfer continuous. Indeed, let $x$ not be a CBE. Then there exists $\widetilde{y} \in \widetilde{X}$ defined by $\widetilde{y}_{j}=1$, for each $j$ and a neighborhood $\mathcal{V}_{x}$ of $x$ such that for each $z \in \mathcal{V}_{x}, F(z, \widetilde{y})>F(z, h(z))$. $F$ is also $h$-diagonal transfer quasiconcave in $\widetilde{y}$. Indeed, for any finite subset $\left\{\widetilde{y}^{1}, \ldots, \widetilde{y}^{p}\right\} \subset \widetilde{X}$, there exists a corresponding finite subset $\left\{x^{1}, \ldots, x^{p}\right\}=\{(a, a, a)\} \subset X$ (with $a=\max _{j, h=1, \ldots, p} \widetilde{y}_{j}^{h}$ ) such that for any subset $J \subset\{1,2, \ldots, p\}$ and any $x \in \operatorname{co}\left\{x^{h}: h \in J\right\}$, we have $\min _{h \in J} F\left(x, \widetilde{y}^{h}\right) \leq F(x, h(x))$. By Theorem 3.4 this game admits a CBE. 


\section{Conclusion}

The main contribution of this paper is to offer existence conditions for CBE, BZE, BVE and BNE in n-player games that satisfy weak continuity and quasi-concavity conditions. We use Lemma 3.1 to derive general necessary and sufficient existence conditions (Theorems 3.1, 3.3, 3.4). Another contribution of the paper is to highlight the relevance of BVE for situational decision-making. BVE depicts players that consider their maximin value as a benchmark in their deliberation. An individually rational player should never follow an equilibrium strategy that does worse than his security level. The resulting conjecture for situational gaming when a game admits both a NE and a $\mathrm{BE}$ is that players would choose $\mathrm{BE}$ if the outcome value is at least equal to the security level, else they would choose NE.

Several research directions are attractive in terms of future work on this topic. The first is additional perspectives on situational gaming. Situational gaming requires deeper scrutiny of other possible behavior rules and equilibrium concepts that complement Nash and Berge. The second avenue for future research concerns mutual support and the question of when BVE is to be applied and when another equilibrium concept is to be applied. Another interesting line of research would be to define classes of games where BVE is Pareto optimal and where BVE is always Pareto dominated.

\section{Appendix A: Proofs}

\section{Proof of Lemma 3.2.}

1) $A$ is a nonempty set. The conditions 1) and 2) of Lemma 3.2 imply that $\forall i \in I, \alpha_{i}=$ $\sup _{x_{i} \in X_{i} y_{-i} \in X_{-i}} u_{i}\left(x_{i}, y_{-i}\right)$ exists. Since the functions $u_{i}, i \in I$, are continuous over the compact $X$, then $\forall i \in I, \exists \widetilde{x}_{i} \in X_{i}$ such that

$$
\alpha_{i}=\sup _{x_{i} \in X_{i}} \inf _{y_{-i} \in X_{-i}} u_{i}\left(x_{i}, y_{-i}\right)=\inf _{y_{-i} \in X_{-i}} u_{i}\left(\widetilde{x}_{i}, y_{-i}\right)
$$

Let $\widetilde{x}=\left(\widetilde{x}_{1}, \ldots, \widetilde{x}_{n}\right) \in X$, we have for all $i \in I, u_{i}(\widetilde{x})=u_{i}\left(\widetilde{x}_{i}, \widetilde{x}_{-i}\right) \geq$ $\inf _{y_{-i} \in X_{-i}} u_{i}\left(\widetilde{x}_{i}, y_{-i}\right)=\alpha_{i}$. Thus $A \neq \emptyset$.

2) $A$ is convex in $X$. Let $\bar{x}$ and $\overline{\bar{x}}$ be two elements in $A$ and let $\lambda \in[0,1]$. We show that $\lambda \bar{x}+(1-\lambda) \overline{\bar{x}} \in A$. Since $\bar{x}$ and $\overline{\bar{x}}$ are two elements in $A, \alpha_{i} \leq u_{i}(\bar{x})$ and $\alpha_{i} \leq u_{i}(\overline{\bar{x}})$, $\forall i \in I$. Hence $\alpha_{i} \leq \min \left\{u_{i}(\bar{x}), u_{i}(\overline{\bar{x}})\right\}$ for each $i \in I$. Since the functions $u_{i}, i \in I$, are quasiconcave over $X, \alpha_{i} \leq u_{i}(\lambda \bar{x}+(1-\lambda) \overline{\bar{x}})$, for each $i \in I$, and $\lambda \in[0,1]$. Therefore, $\lambda \bar{x}+(1-\lambda) \overline{\bar{x}} \in A$.

3) $A$ is compact in $X$. Since $X$ is compact, it is sufficient to prove that $A$ is closed. Let $\left\{x^{p}\right\}_{p \geq 1}$ be a net of elements in $A$ converging to $\bar{x}$. We show that $\bar{x} \in A$. We have $\forall p \geq 1, x^{p} \in A$, 
then

$$
\forall p \geq 1, \forall i \in I, \alpha_{i} \leq u_{i}\left(x^{p}\right)
$$

Taking into account condition 1) and the continuity of $u_{i}$ of Lemma 3.2, when $p \rightarrow \infty$, we obtain: $\forall i \in I, \alpha_{i} \leq u_{i}(\bar{x})$, which means that $\bar{x} \in A$.

\section{Proof of Lemma 3.3.}

Sufficiency. Let $\bar{x} \in A$ such that $\max _{\widehat{y} \in \widehat{X}} \Gamma(\bar{x}, \widehat{y})=\Gamma(\bar{x}, g(\bar{x}))$, this equality implies $\forall \widehat{y} \in \widehat{X}$, $\Gamma(\bar{x}, \widehat{y})-\Gamma(\bar{x}, g(\bar{x}))=\sum_{i \in I}\left(u_{i}\left(\bar{x}_{i}, \widehat{y}_{-i}\right)-u_{i}(\bar{x})\right) \leq 0$. For an arbitrarily fixed $i \in I$, we have $\forall \widehat{y} \in \widehat{X}, \Gamma(\bar{x}, \widehat{y})=\left(u_{i}\left(\bar{x}_{i}, \widehat{y}_{-i}\right)-u_{i}(\bar{x})\right)+\sum_{\substack{j \neq i \\ j \in I}}\left(u_{j}\left(\bar{x}_{j}, \widehat{y}_{-j}\right)-u_{j}(\bar{x})\right) \leq 0$.

For $\widehat{y} \in \widehat{X}$ such that $\widehat{y}_{-i}$ is arbitrarily chosen in $X_{-i}$ and $\widehat{y}_{-j}=\bar{x}_{-j}, \forall j \neq i$, we have $\sum_{\substack{j \neq i \\ j \in I}}\left(u_{j}\left(\bar{x}_{j}, \widehat{y}_{-j}\right)-u_{j}(\bar{x})\right)=0$. From the last inequality, we deduce that $\forall \widehat{y}_{-i} \in X_{-i}$, $u_{i}\left(\bar{x}_{i}, \widehat{y}_{-i}\right) \leq u_{i}(\bar{x})$. Since $i$ is arbitrarily chosen in $I$, we have $\forall i \in I, \forall y_{-i} \in X_{-i}$, $u_{i}\left(\bar{x}_{i}, y_{-i}\right) \leq u_{i}(\bar{x})$. Taking into account the fact that $\bar{x} \in A$, we deduce that $\bar{x}$ is a BVE of the game (2.1).

Necessity. Let $\bar{x} \in X$ be a BVE of the game (2.1). The second condition of Definition 2.3 implies that $\bar{x} \in A$. The first condition of Definition 2.3 implies $u_{i}\left(\bar{x}_{i}, t_{-i}\right) \leq u_{i}(\bar{x})$, $\forall t_{-i} \in X_{-i}, \forall i \in I$, hence $\Gamma(\bar{x}, \widehat{y})-\Gamma(\bar{x}, g(\bar{x}))=\sum_{i \in I}\left(u_{i}\left(\bar{x}_{i}, \widehat{y}_{-i}\right)-u_{i}(\bar{x})\right) \leq 0, \forall \widehat{y} \in \widehat{X}$, i.e. $\max _{\widehat{y} \in \widehat{X}} \Gamma(\bar{x}, \widehat{y}) \leq \Gamma(\bar{x}, g(\bar{x}))$. Taking into account Remark 3.1, we obtain $\max _{\widehat{y} \in \widehat{X}} \Gamma(\bar{x}, \widehat{y})=$ $\Gamma(\bar{x}, g(\bar{x}))$.

\section{Proof of Lemma 3.4.}

1) $A^{\prime}$ is nonempty. Because function $u_{i}$ is bounded, $\alpha_{i}$ exists for each $i \in I$. The function $x_{i} \mapsto$ $\inf _{x_{-i} \in X_{-i}} u_{i}\left(x_{i}, x_{-i}\right)$ is upper semicontinuous on the compact $X_{i}$. There exists therefore $\bar{x}_{i} \in X_{i}$ such that

$$
\inf _{x_{-i} \in X_{-i}} u_{i}\left(\bar{x}_{i}, x_{-i}\right) \geq \alpha_{i} .
$$

Hence, $\bar{x}=\left(\bar{x}_{1}, \ldots, \bar{x}_{n}\right)$ is an element of $A^{\prime}$.

2) By quasiconcavity of $\inf _{x_{-i} \in X_{-i}} u_{i}\left(x_{i}, x_{-i}\right)$ in $x_{i}, A^{\prime}$ is convex. 
3) $A^{\prime}$ is compact in $X$. Since $X$ is compact, it is sufficient to prove that $A^{\prime}$ is closed. Let $\left\{x^{p}\right\}_{p \geq 1}$ be a set of elements of $A^{\prime}$ converging to $\bar{x}$. We show that $\bar{x} \in A^{\prime}$. We have $\forall p \geq 1, x^{p} \in A$, then

$$
\forall p \geq 1, \forall i \in I, \alpha_{i} \leq \inf _{x_{-i} \in X_{-i}} u_{i}\left(x_{i}^{p}, x_{-i}\right)
$$

Since $x_{i} \mapsto \inf _{x_{-i} \in X_{-i}} u_{i}\left(x_{i}, x_{-i}\right)$ is upper semicontinuous on the compact $X_{i}, \forall i \in I, \alpha_{i} \leq$ $\inf _{x_{-i} \in X_{-i}} u_{i}\left(\bar{x}_{i}, x_{-i}\right)$, i.e. $\bar{x} \in A^{\prime}$.

\section{Proof of Lemma 3.5.}

Sufficiency. Suppose that $\sup _{(\widehat{y}, z) \in \widehat{X} \times X} \widetilde{\Gamma}(\bar{x},(\widehat{y}, z))=\widetilde{\Gamma}(\bar{x}, \widetilde{g}(\bar{x}))$, i.e.

$$
\sum_{i \in I}\left(u_{i}\left(\bar{x}_{i}, y_{-i}\right)+u_{i}\left(\bar{x}_{-i}, z_{i}\right)\right) \leq \sum_{i \in I}\left(u_{i}(\bar{x})+u_{i}(\bar{x})\right), \forall(\widehat{y}, z) \in \widehat{X} \times X
$$

If we take $y_{-i}=\bar{x}_{-i}, \forall i \in I$ in (4.1), we conclude that $\sum_{i \in I} u_{i}\left(\bar{x}_{-i}, z_{i}\right) \leq \sum_{i \in I} u_{i}(\bar{x}), \forall z \in X$, which implies that $\bar{x}$ is Nash equilibrium of the game (2.1).

If we take $z=\bar{x}$ in equation (4.1), we conclude that $\bar{x}$ satisfies the property 1 ) of Definition 2.5 and since $\bar{x}$ is a Nash equilibrium, it is also individually rational. We conclude that $\bar{x}$ is a BVE of the game (2.1).

Necessity. Suppose that $\bar{x}$ is a BNE of the game (2.1). The fact that $\bar{x}$ is a NE of the game (2.1) implies

$$
\max _{z \in X} \sum_{i \in I} u_{i}\left(\bar{x}_{-i}, z_{i}\right)=\sum_{i \in I} u_{i}(\bar{x}) .
$$

The fact that $\bar{x}$ is a BVE of the game (2.1) implies

$$
\max _{\widehat{y} \in \widehat{X}} \sum_{i \in I} u_{i}\left(\bar{x}_{i}, y_{-i}\right)=\sum_{i \in I} u_{i}(\bar{x})
$$

The two equalities (4.2) and (4.3) imply $\max _{(\widehat{y}, z) \in \widehat{X} \times X} \widetilde{\Gamma}(\bar{x},(\widehat{y}, z))=\widetilde{\Gamma}(\bar{x}, \widetilde{g}(\bar{x}))$.

\section{Proof of Lemma 3.6.}

Sufficiency. Let $\bar{x} \in X$ such that $\sup F(\bar{x}, \widetilde{y})=0$, this equality implies $\forall \widetilde{y} \in \widetilde{X}, F(\bar{x}, \widetilde{y})=$

$$
\widetilde{y} \in \widetilde{X}
$$

$\sum_{m \in M} \sum_{j \in R_{m}}\left(u_{j}\left(\bar{x}_{-S_{m}}, y_{S_{m}}\right)-u_{j}(\bar{x})\right) \leq 0$. For an arbitrarily fixed $m_{0} \in M, j_{0} \in R_{m_{0}}$, we 
have $\forall \widetilde{y} \in \widetilde{X}$,

$$
\begin{aligned}
F(\bar{x}, \widetilde{y})= & u_{j_{0}}\left(\bar{x}_{-S_{m_{0}}}, y_{S_{m_{0}}}\right)-u_{j_{0}}(\bar{x})+\sum_{j \in R_{m_{0}} /\left\{j_{0}\right\}}\left(u_{j}\left(\bar{x}_{-S_{m}}, y_{S_{m}}^{j_{m}}\right)-u_{j}(\bar{x})\right) \\
& +\sum_{m \in M /\left\{m_{0}\right\}} \sum_{j \in R_{m}}\left(u_{j}\left(\bar{x}_{-S_{m}}, y_{S_{m}}^{j_{m}}\right)-u_{j}(\bar{x})\right) \\
& \leq 0 .
\end{aligned}
$$

For $\tilde{y} \in \tilde{X}$ such that $y_{S_{m_{0}}}$ is arbitrarily chosen in $\tilde{X}_{S_{m_{0}}}$ and $y_{S_{m}}^{j_{m}}=$ $\bar{x}_{S_{m}}, \forall m \neq m_{0}, \quad j_{m} \neq j_{0}$, we obtain $\sum_{j \in R_{m_{0}} /\left\{j_{0}\right\}}\left(u_{j}\left(\bar{x}_{-S_{m}}, y_{S_{m}}^{j_{m}}\right)-u_{j}(\bar{x})\right)+$ $\sum_{m \in M /\left\{m_{0}\right\}} \sum_{j \in R_{m}}\left(u_{j}\left(\bar{x}_{-S_{m}}, y_{S_{m}}^{j_{m}}\right)-u_{j}(\bar{x})\right)=0$. From the last inequality, we deduce that $\forall y_{S_{m_{0}}} \in X_{S_{m_{0}}}, u_{j_{0}}\left(\bar{x}_{-S_{m_{0}}}, y_{S_{m_{0}}}\right) \leq u_{j_{0}}(\bar{x})$. Since $m_{0} \in M$ and $j_{0} \in R_{m_{0}}$ are arbitrarily chosen, then $\bar{x}$ is a CBE of the game (2.1).

Necessity. Let $\bar{x}$ be a CBE of the game (2.1). Then, for each $\widetilde{y} \in \widetilde{X}$,

$$
F(\bar{x}, \widetilde{y})=\sum_{m \in M} \sum_{j \in R_{m}}\left(u_{j}\left(\bar{x}_{-S_{m}}, y_{S_{m}}\right)-u_{j}(\bar{x})\right) \leq 0
$$

By the construction of $F$, we have $\sup _{\widetilde{X}} F(x, \widetilde{y}) \geq 0$, for each $x \in X$. We conclude that $\sup _{\widetilde{x}} F(\bar{x}, \widetilde{y})=0$. $\widetilde{y} \in \widetilde{X}$

\section{Appendix B: Computational Complexity}

In what follows, we provide with preliminary results for computational complexity of BZE, BVE and BNE in two-player games by considering the correspondance between Berge and Nash notions.

For each player $i \in I$ in the game $G$, define a two player subgame

$$
G_{i}=\left\langle\left\{1_{i}, 2_{i}\right\}, X_{i}, X_{-i}, g_{1}^{i}, g_{2}^{i}\right\rangle
$$

Player $1_{i}$ 's strategy set is $X_{i}$ and the strategy set of player $2_{i}$ is $X_{-i}$. If player $1_{i}$ chooses $x_{i}$ and $2_{i}$ play $x_{-i}$, then the payoffs are $g_{1}^{i}\left(x_{i}, x_{-i}\right)=\sum_{j \in-i} u_{j}(x), g_{2}^{i}\left(x_{i}, x_{-i}\right)=u_{i}(x)$.

We have the following lemma.

LEMMA 4.1 The strategy profile $\bar{x} \in X$ is a BZE of the game (2.1) if and only if for each player $i \in I, \bar{x}=\left(\bar{x}_{i}, \bar{x}_{-i}\right)$ is a Nash equilibrium of the subgame (4.4).

\section{Proof.}


Necessity: Let $\bar{x} \in X$ be a Berge-Zhukovskii equilibrium of the game (2.1). Then, for each player $i$, and $y_{-i} \in X_{-i}$, we have

$$
u_{i}\left(\bar{x}_{i}, y_{-i}\right) \leq u_{i}(\bar{x})
$$

Let $i \in I$ be any player, then for each $j \in-i$, we have for each $y_{i} \in X_{i}$,

$$
u_{j}\left(\bar{x}_{j}, y_{i}, \bar{x}_{-\{i, j\}}\right)=u_{j}\left(y_{i}, \bar{x}_{-i}\right) \leq u_{j}(\bar{x})
$$

Therefore, for each $y_{i} \in X_{i}$, we have

$$
g_{1}^{i}\left(y_{i}, \bar{x}_{-i}\right) \leq g_{1}^{i}(\bar{x})
$$

Then by (4.5) and (4.6), the strategy $\bar{x}$ is a Nash equilibrium for the subgame (4.4).

Sufficiency: Let $\bar{x}$ be any strategy in $X$ such that for each player $i, \bar{x}=\left(\bar{x}_{i}, \bar{x}_{-i}\right)$ is a Nash equilibrium of the subgame (4.4). Then for each player $i$, and for each $y_{i} \in X_{i}, y_{-i} \in X_{-i}$, we have

$$
g_{1}^{i}\left(y_{i}, \bar{x}_{-i}\right) \leq g_{1}^{i}(\bar{x}) \text { and } g_{2}^{i}\left(\bar{x}_{i}, y_{-i}\right) \leq g_{2}^{i}(\bar{x})
$$

By (4.7) and definition of $g_{2}^{i}$, we obtain for each $y_{-i} \in X_{-i}, u_{i}\left(\bar{x}_{i}, y_{-i}\right) \leq u_{i}(\bar{x})$, i.e. $\bar{x} \in X$ is a Berge-Zhukovskii equilibrium for the game (2.1).

By Lemma 4.1, we deduce the following corollary.

Corollary 4.1 The strategy profile $\bar{x} \in X$ is a BVE of the game (2.1) if and only if for each player $i \in I, \bar{x}=\left(\bar{x}_{i}, \bar{x}_{-i}\right)$ is a Nash equilibrium of the subgame (4.4) and $\bar{x} \in A$.

REMARK 4.1 Lemma 4.1 and Corollary 4.1 can be extended to mixed strategies.

Daskalakis et al. (2009) show that the computation of Nash equilibrium is PPADcomplete. Since BNE is a Nash equilibrium, we deduce that it pertains to the class of problems that are PPAD-complete. Furthermore, combining Papadimitriou et al.'s result with Lemma 4.1,Corollary 4.1 and Remark 4.1, we conclude that the computation of BZE and BVE is PPAD-complete.

\section{References}

Abalo, K., Kostreva, M. (1996): Equi-well-posed Games. Journal of Optimization Theory and Applications, 89, 89-99. 
Abalo, K., Kostreva, M. (2004): Some existence theorems of Nash and Berge equilibria. Applied Mathematics Letters, 17, 569-573.

Baye, M.R., Tian, G., Zhou, J. (1993): Characterizations of the existence of equilibria in games with discontinuous and Non-Quasiconcave Payoffs. The Review of Economic Studies, 60, 935-948.

Berge, C. (1957): Théorie générale des jeux à n-personnes. Paris, Gauthier Villars.

Colman, A.M., Körner, T.W., Musy, O., TAZdä̈T, T. (2011): Mutual support in games: some properties of Berge equilibria. Journal of Mathematical Psychology, 55, 166-175.

Courtois, P., Nessah, R., TAzdaït, T. (2015): How to play games? Nash versus Berge equilibria. Economics and Philosophy, 31(1), 123-139.

Crettez, B. (2016): A new sufficient condition for a Berge equilibrium to be a Berge-Vaisman equilibrium. Journal of Quantitative Economics, DOI 10.1007/s40953-016-0066-z

Daskalakis, C., Goldberg, P.W., Papadimitriou, C. H (2009): The complexity of computing a Nash equilibrium. SIAM Journal on Computing, 39(1), 195-259.

FAN, K. (1972): A minimax inequality and application, in: O. Shisha (Ed.), Inequalities, III, New York, Academic Press.

Kreps, D.M. (1990): Corporate culture and economic theory? in: J.E. Alt and K.A Shepsle (Eds), Perspectives on Positive Political Economy, Cambridge, Cambridge University Press, 90-143.

Larbani, M., Nessah, R. (2008): A note on the existence of Berge and Berge-Nash equilibria, Mathematical Social Sciences, 55, 258-271.

Nessah, R., LARbani, M. (2004): g-Maximum equality. In: Takahashi,W., Tanaka, T. (eds.) Nonlinear Analysis and Convex Analysis, Yokohama Publishers, Yokohama pp. 391-400.

Nessah, R., LARbani, M. (2014): Berge-Zhukovskii equilibria: existence and characterization. International Game Theory Review, 16, 1450012.

Nessah, R., Larbani, M., Tazdaït, T. (2007): A note on Berge equilibrium. Applied Mathematics Letters, 20, 926-932.

Nessah R., LARbani M., TAZDAit T. (2009): New sufficient conditions for the g-maximum inequality. In: Takahashi, W., Tanaka, T.(Eds.), Nonlinear Analysis and Convex Analysis, Yokohama Publishers, Yokohama, pp. 151-160

NeSSAH, R., TAZDAÏT, T. (2013): Absolute optimal solution for a compact and convex game. European Journal of Operational Research, 224, 353-361.

Nessah, R. AND TIAN, G. (2013): Existence of solution of minimax inequalities, equilibria in games and fixed points without convexity and compactness assumptions. Journal of Optimization Theory and Applications, 157, 75-95 
Rousseau, J.J. (1755/1964): Discours sur l'origine et les fondements de l'inégalité parmi les hommes. La Pléiade, Tome 3, Paris, Galimard.

TiAN, G., ZHOU, Z. (1993): Quasi-Inequalities without the concavity assumption. Journal of Mathematical Analysis and Applications 172, 289-299.

Vaisman, K.S. (1994): Berge equilibrium in one differential game. In: Complex Dynamical Systems. Pskov, Pskov Pedagogical Institute, 58-63. (in Russian)

VAISMAn, K.S. (1995): Berge equilibrium. Phd Thesis. Saint Petersburg University. (in Russian)

ZhukovskiI, V.I. (1985): Some problems of non-antagonistic differential games. In P. Kenderov (Ed.), Matematiceskie Metody v Issledovanii Operacij [Mathematical Methods in Operations Research]. Sofia, Bulgarian Academy of Sciences, 103-195.

ZhukovskiI, V.I., ChikriI, A.A. (1994): Linear-quadratic differential games. Kiev, Naukova Dumka. (in Russian) 